\title{
Blood Flow Restriction Therapy Versus Standard Care for Reducing Quadriceps Atrophy After Anterior Cruciate Ligament Reconstruction
}

\author{
Lauren Anne Lipker, Caitlyn Rae Persinger, Bradley Steven Michalko, and Christopher J. Durall
}

\begin{abstract}
Clinical Scenario: Quadriceps atrophy and weakness are common after anterior cruciate ligament reconstruction (ACLR). Blood flow restriction (BFR) therapy, alone or in combination with exercise, has shown some promise in promoting muscular hypertrophy. This review was conducted to ascertain the extent to which current evidence supports the use of BFR for reducing quadriceps atrophy following ACLR in comparison with standard care. Clinical Question: Is BFR more effective than standard care for reducing quadriceps atrophy after ACLR? Summary of Key Findings: The literature was searched for studies that directly compared BFR treatment to standard care in patients with ACLR. Three level I randomized control trial studies retrieved from the literature search met the inclusion criteria. Clinical Bottom Line: Reviewed data suggest that a short duration (13 d) of moderate-pressure BFR combined with low-resistance muscular training does not appear to measurably affect quadriceps cross-sectional area. However, a relatively long duration (15 wk) of moderate-pressure BFR combined with lowresistance muscular training may increase quadriceps cross-sectional area to a greater extent than low-resistance muscular training alone. The results of the third randomized control trial suggest that employing BFR while immobilized in the early postoperative period may reduce quadriceps atrophy following ACLR. Additional data are needed to establish if the benefits of BFR on quadriceps atrophy after ACLR outweigh the inherent risks and costs. Strength of Recommendation: All evidence for this review was level 1 (randomized control trial) based on the Centre for Evidence-Based Medicine criteria. However, the findings were inconsistent across the 3 studies regarding the effects of BFR on quadriceps atrophy resulting in a grade "B" strength of recommendation.
\end{abstract}

Keywords: knee-extensor strength, postoperative rehabilitation, vascular occlusion

\section{Clinical Scenario}

Quadriceps atrophy and weakness are common after anterior cruciate ligament reconstruction (ACLR). ${ }^{1,2}$ Blood flow restriction (BFR) therapy, alone or in combination with exercise, has shown some promise in promoting muscular hypertrophy. ${ }^{1-3}$ This review was conducted to ascertain the extent to which current evidence supports the use of BFR to reduce quadriceps muscle atrophy following ACLR in comparison with standard care.

\section{Focused Clinical Question}

Is BFR more effective than standard care for reducing quadriceps atrophy after ACLR?

\section{Summary of Search, "Best Evidence" Appraised, and Key Findings}

- The literature was searched for studies that directly compared BFR to standard care in patients with ACLR.

- Three level 1 randomized clinical trial (RCT) studies were retrieved. ${ }^{1,2,4}$

Lipker, Persinger, Michalko, and Durall are with the University of Wisconsin-La Crosse, La Crosse, WI, USA. Durall is also with Student Health Center, Physical Therapy Unit, University of Wisconsin-La Crosse, La Crosse, WI, USA. Persinger (caitlynpersinger@gmail.com) is corresponding author.
- One RCT showed greater improvements in quadriceps crosssectional area (CSA) after 15 weeks of low-resistance muscular training with moderate-pressure BFR $(180 \mathrm{~mm} \mathrm{Hg})$ compared with low-resistance muscular training alone. ${ }^{2}$

- A second study found that 13 days of low-resistance muscular training with moderate-pressure (130-180 mm Hg) BFR resulted in no difference in quadriceps CSA reduction versus low-resistance muscular training alone. ${ }^{4}$

- The third study reported that 10 days of moderate-pressure (180-260 mm Hg) intermittent BFR while immobilized in a knee brace from the third to 14th postoperative days (standard care at the study site) resulted in less quadriceps CSA reduction than sham BFR with knee immobilization. ${ }^{1}$

\section{Clinical Bottom Line}

Reviewed data suggest that a short duration (13 d) of moderatepressure BFR combined with low-resistance muscular training does not appear to measurably affect quadriceps CSA. ${ }^{4}$ However, a relatively long duration (15 wk) of moderate-pressure BFR combined with low-resistance muscular training may increase quadriceps CSA to a greater extent than low-resistance muscular training alone. ${ }^{2}$ The results of the third RCT suggest that employing BFR while immobilized in the early postoperative period may reduce quadriceps atrophy following ACLR. ${ }^{1}$ Additional data are needed to establish if the benefits of BFR on quadriceps atrophy after ACLR outweigh the inherent risks and costs. 


\section{Strength of Recommendation}

All 3 reviewed studies were level 1 RCTs., ${ }^{1,2,4}$ However, the findings were inconsistent across the 3 studies regarding the effects of BFR on quadriceps atrophy resulting in a grade "B" strength of recommendation. ${ }^{1,2,4}$

\section{Search Strategy}

\section{Terms Used to Guide Search Strategy}

- Patient/Client Group: anterior cruciate ligament reconstruction

- Intervention: blood flow restriction training OR occlusive training OR vascular restriction rehabilitation AND (therapeutic or treatment)

- Comparison: traditional rehabilitation (strengthening OR resistance)

- Outcome(s): cross-sectional area of knee extensors OR atrophy OR knee extensor torque

\section{Sources of Evidence Searched}

- PubMed

- CINAHL Plus

- SPORTDiscus

- MEDLINE

- EBSCOhost

- Cochrane Database of Systematic Reviews

- Additional resources obtained via manual search of reference lists

\section{Inclusion and Exclusion Criteria}

\section{Inclusion Criteria}

- Patients who underwent ACLR

- Studies that compared BFR training to standard care

- Limited to humans

- Limited to English language

- Limited to 2000-2018 publication dates

\section{Exclusion Criteria}

- Case studies

- Studies involving participants who had other pathologies of the knee in addition to ACLR

\section{Results of Search}

An extensive search of the literature yielded many articles; however, only 3 studies met the eligibility requirements and were considered for review. ${ }^{1,2,4}$ These studies are summarized in Table 1. Two studies were RCTs that examined the effect of BFR with exercise versus low-resistance exercise alone after ACLR. ${ }^{2,4}$ One study was an RCT that examined the effect of BFR while immobilized in a knee brace compared with a sham treatment, where the cuff was applied but not inflated during knee brace immobilization. ${ }^{1}$

\section{Best Evidence}

The studies identified in Table 1 are the best available evidence found for this review. All 3 articles were level 1 evidence RCTs based on Centre for Evidence-Based Medicine (2011). ${ }^{5}$

\section{Implications for Practice, Education, and Future Research}

The objective of this review was to determine if there is evidential support for BFR to reduce quadriceps atrophy following ACLR. The 3 studies identified as the best available evidence to answer this question were high-quality RCTs, but their outcomes were mixed. Two studies reported a significant reduction in quadriceps atrophy in comparison with their control groups, whereas the third study found no between-group differences in atrophic changes. ${ }^{1,2,4}$ This disparity in outcomes may be due to a variety of factors including but not limited to, cuff size, occlusive pressure, individual treatment session duration, and length of the intervention period. For instance, Iversen et $\mathrm{al}^{4}$ used occlusive inflation pressures ranging from 130 to $180 \mathrm{~mm} \mathrm{Hg}$, whereas Takarada et $\mathrm{al}^{1}$ used occlusive pressures ranging from 180 to $260 \mathrm{~mm} \mathrm{Hg}$. Ohta et $\mathrm{al}^{2}$ used an occlusive inflation pressure of $180 \mathrm{~mm} \mathrm{Hg}$ exclusively. Both Takarda et $\mathrm{al}^{1}$ and Ohta et $\mathrm{al}^{2}$ reported benefits with BFR suggesting that higher inflation pressures may be required to significantly affect muscle CSA. The tourniquet cuff size also varied between studies. Iversen et $\mathrm{al}^{4}$ used a $14-\mathrm{cm}$ pneumatic cuff, whereas Takarada et $\mathrm{al}^{1}$ used a 9 -cm cuff for their study. Ohta et $\mathrm{al}^{2} \mathrm{did}$ not report the dimensions of the tourniquet cuff used in their study.

The length of the individual treatment sessions varied somewhat between the studies as did the intervention period duration. Ohta et $\mathrm{al}^{2}$ used BFR for as long as each participant could endure during the exercise sessions with no specific inflation duration time per day, whereas subjects in both the Iversen et $\mathrm{al}^{4}$ and Takarada et $\mathrm{al}^{1}$ studies had BFR for 50 minutes per day. Given these differences and the disparity in outcomes between these studies, it is difficult to make recommendations regarding the optimal duration of individual BFR treatment sessions for retarding quadriceps atrophy after ACLR.

The length of the intervention period also varied between studies. Ohta et $\mathrm{al}^{2}$ studied the effects of a 15 -week intervention period, whereas the intervention period in the Iversen et $\mathrm{al}^{4}$ and Takarada et $\mathrm{al}^{1}$ investigations were 13 and 10 days, respectively. Ohta et $\mathrm{al}^{2}$ and Iverson et $\mathrm{al}^{4}$ both used BFR with low-resistance exercise, although only Ohta et $\mathrm{al}^{2}$ reported a benefit with BFR suggesting that a relatively lengthy training period may be required for the beneficial effects of BFR on quadriceps CSA to manifest when combined with low-resistance muscular training. There is need for additional data on the impact of treatment session duration (eg, 30, $60 \mathrm{~min}$ ) and intervention period duration (eg, 3, 4, 6, 12, and $14 \mathrm{w}$ ) with BFR after ACLR.

It is important to note that the studies in this review evaluated quadriceps CSA via magnetic resonance imaging (MRI) at different times relative to surgery. Iversen et $\mathrm{al}^{4}$ analyzed MRI data of quadriceps CSA 2 days prior to surgery and 16 days after surgery, whereas Ohta et $\mathrm{al}^{2}$ used MRI data at an unspecified time before surgery and at 16 weeks after surgery. By contrast, Takarada et $\mathrm{al}^{1}$ assessed quadriceps CSA via MRI on the third postoperative day and 12 days later. As baseline quadriceps CSA measurements were taken at disparate time points in these studies, it is difficult to make comparisons. In addition, the anatomic location of quadriceps CSA 


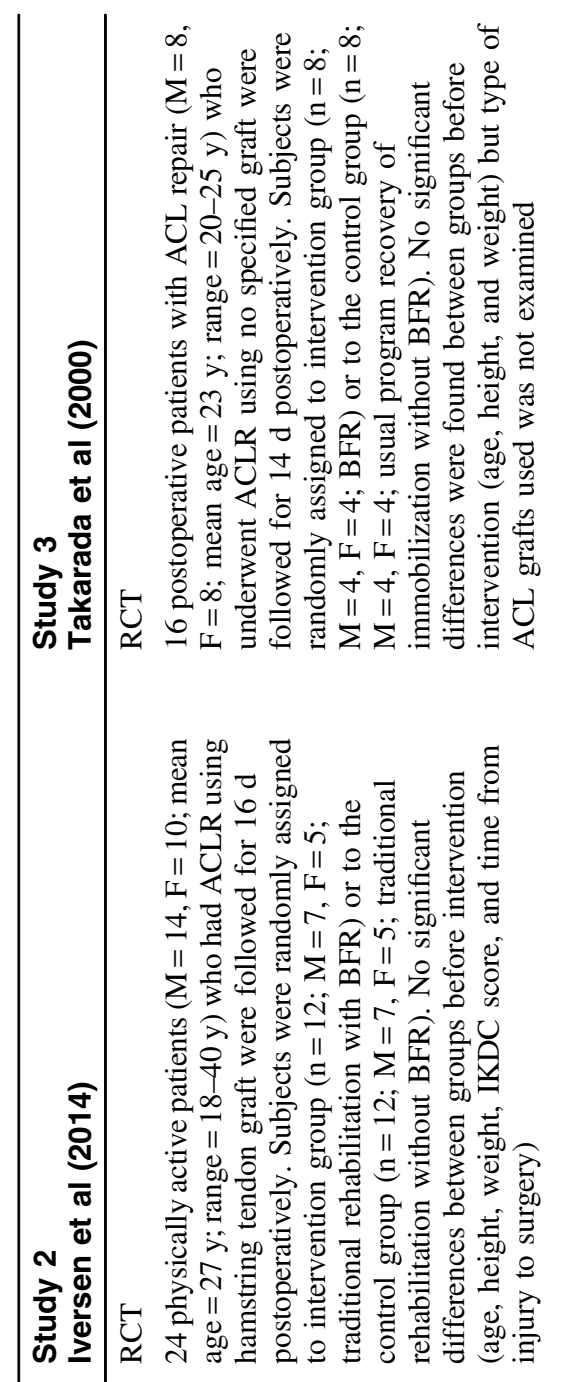

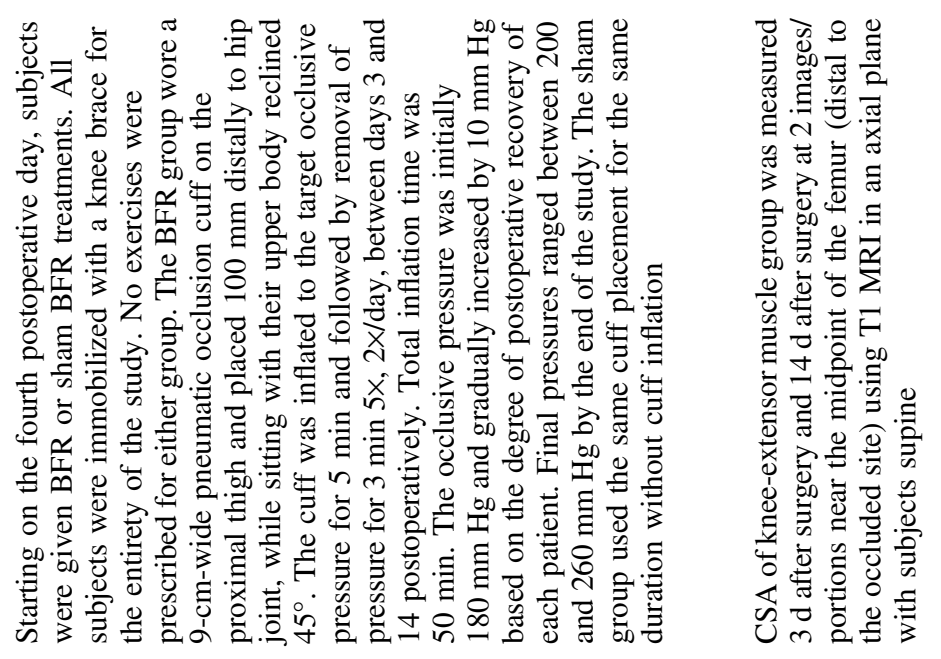

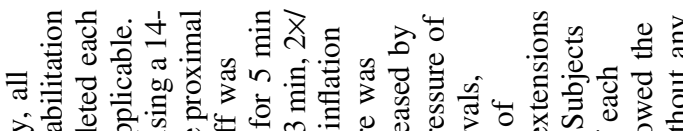

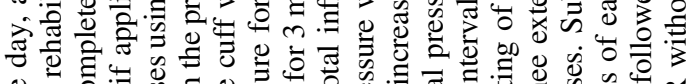

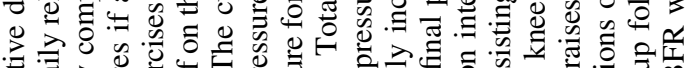

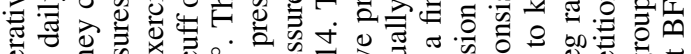

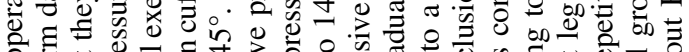

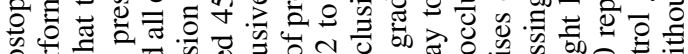

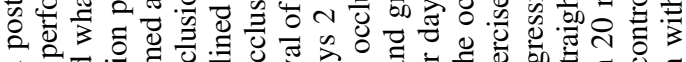

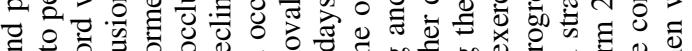

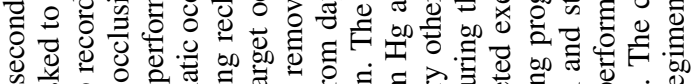

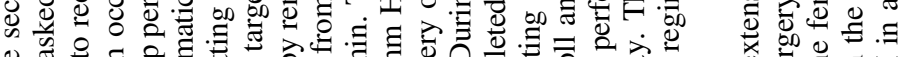

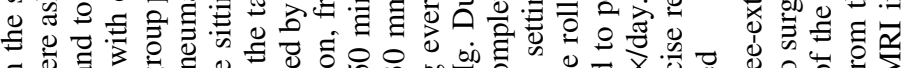
б on 0

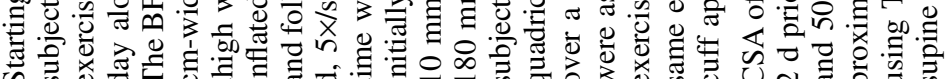

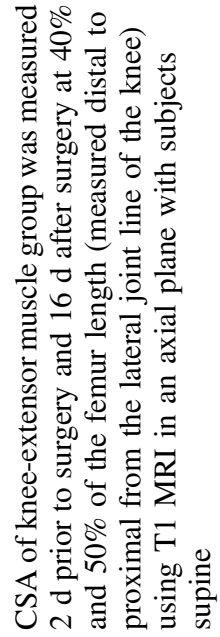

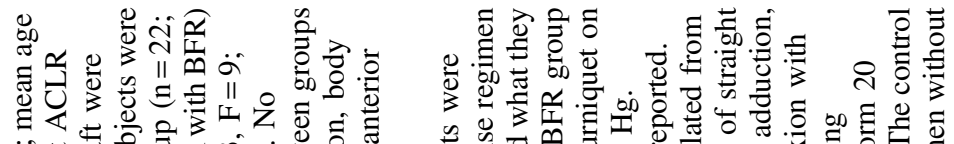
a II 山 认ิ

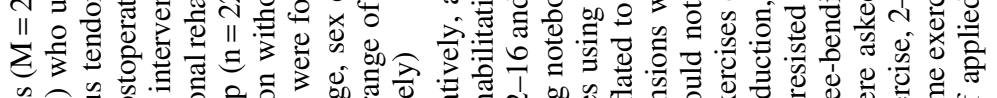

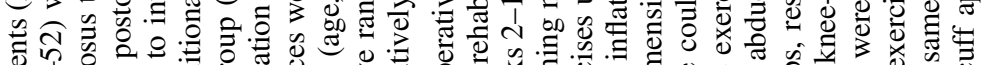

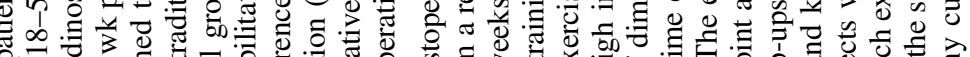
구으.

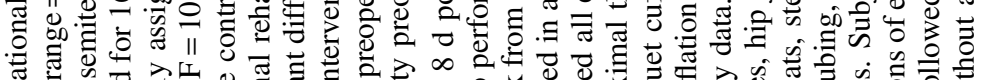

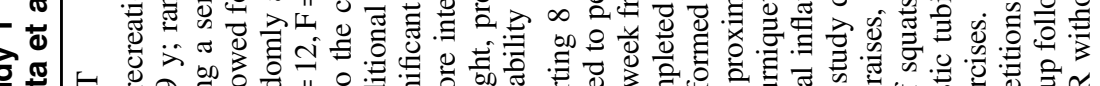

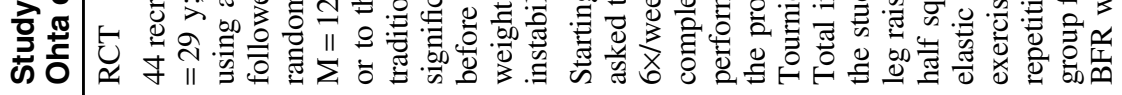

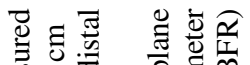

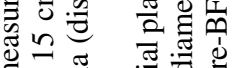

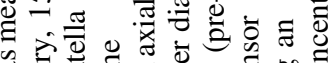

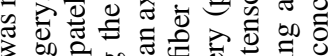

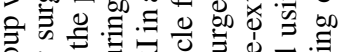

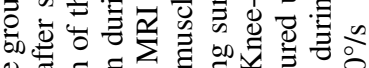
다.

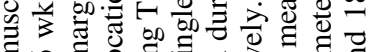

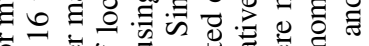

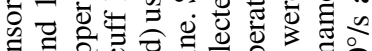

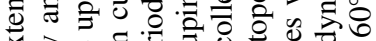

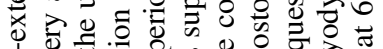

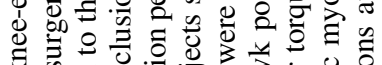

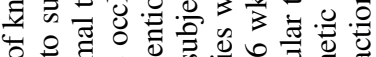

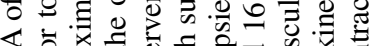

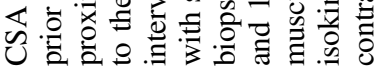
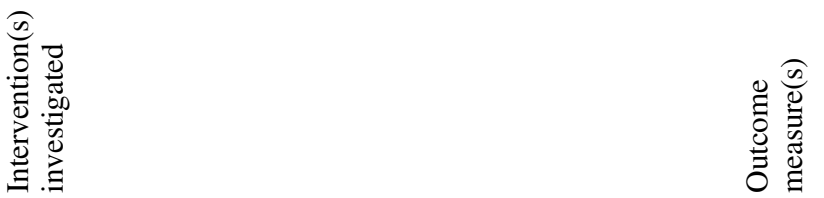


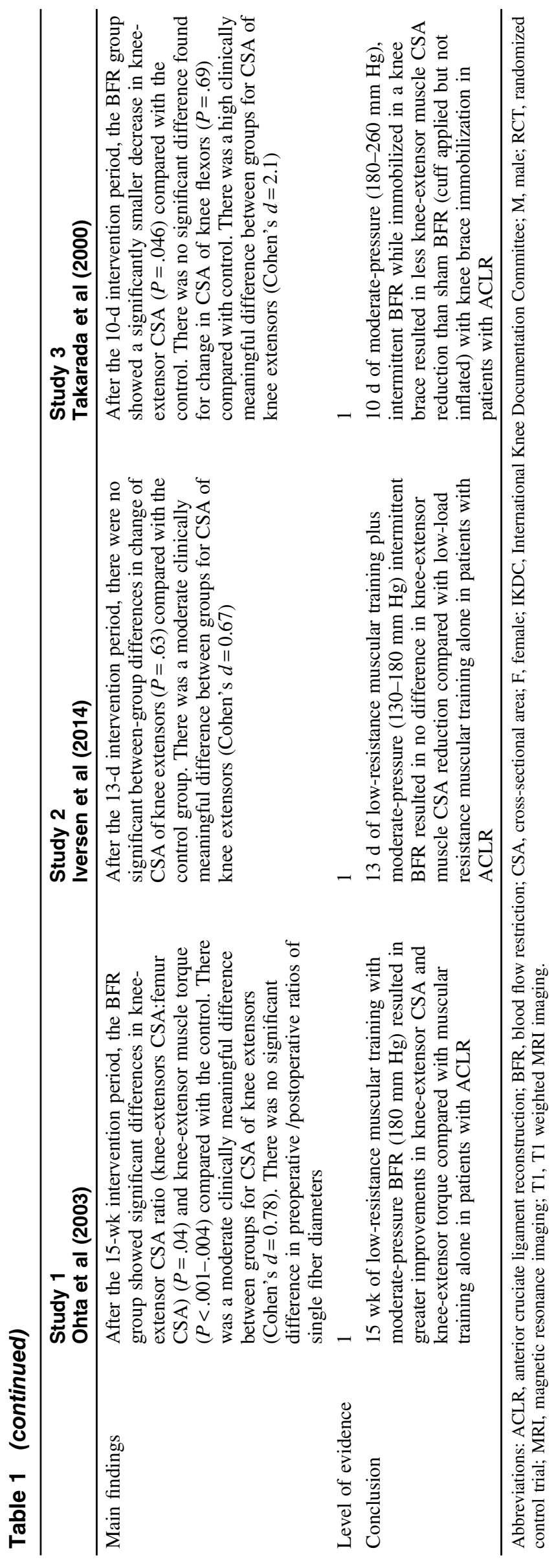


measurements varied between all 3 studies. Iversen et $\mathrm{al}^{4}$ measured quadriceps CSA at $40 \%$ and $50 \%$ of the length of the femur (starting at the distal end), whereas Takarada et $\mathrm{al}^{1}$ measured 2 sections near the midportion of the femur and distal to where BFR was applied. Ohta et $\mathrm{al}^{2}$ expressed MRI results as a ratio of quadriceps CSA to the CSA of the femur, whereas Iverson et al and Takarada et al reported MRI results as the anatomical CSA of the quadriceps alone, further adding to the difficulty of making comparisons between the studies. Future researchers should consider measuring muscle volume rather than CSA as the former may be more accurate for evaluating muscle size. ${ }^{4}$

Future researchers should also consider measuring muscular torque or force output before and after BFR. Only 1 study in this review evaluated knee-extensor and knee-flexor torques. ${ }^{2}$ Ohta et $\mathrm{al}^{2}$ reported significant improvements in both knee torques in the BFR group when compared with the non-BFR treatment group. ${ }^{2}$ The consistent inclusion of torque or force measurements would add a clinically meaningful variable to future research on the effectiveness of BFR therapy.

The mechanisms whereby mechanical tension and metabolic stress from BFR influence muscle CSA are not fully understood at this time. ${ }^{3}$ Restricting blood flow induces local hypoxia, which in turn is thought to cause blood pooling and the accumulation of metabolic subproducts leading to specific nervous and hormonal responses. These responses can result in increased plasma concentration of adrenaline and growth hormone and may reveal the underlying atrophy-attenuating effect from BFR. ${ }^{1}$ In addition, when exercising muscle is exposed to a hypoxic environment, as was the case in 2 of the reviewed studies, the type I muscle fibers that require large amounts of oxygen for contraction cannot adequately function resulting in preferential activation of type 2 muscle fibers. ${ }^{6}$ Rehabilitation following ACLR traditionally involves moderate- to high-intensity ( $>50-60 \%$ 1-repetition maximum) strengthening exercises to facilitate muscle growth. ${ }^{3}$ However, preferential recruitment of type 2 muscle fibers during BFR may reduce postoperative muscle atrophy with low-resistance exercise to a similar extent as high-resistance exercises. ${ }^{3}$ It is theorized that exercise performed during BFR promotes fast-twitch type 2 muscle fiber recruitment and increased stimulation of muscle growth factors. ${ }^{3}$ Increased recruitment has been shown to cause stronger muscle contractions leading to ischemia followed by reactive hyperemia. ${ }^{7}$ However, Ohta et $\mathrm{al}^{2}$ found no significant differences between type 1 and type 2 fiber ratios preintervention and postintervention via muscle biopsies. ${ }^{2}$ Furthermore, there was no preferential increase in diameter for either fiber type. ${ }^{2}$ Future studies that help elucidate how an occlusive stimulus can aid muscle hypertrophy will help in determining the optimal parameters for BFR after ACLR.

The possibility of short- and long-term side effects from BFR merits further investigation. Participants in the study by Ohta et $\mathrm{al}^{2}$ reported a dull pain/discomfort from the tourniquet after roughly 12 minutes of BFR. ${ }^{2}$ The authors of the other 2 reviewed studies did not report adverse effects from the BFR treatment protocols.
In healthy individuals, the impact of BFR on the cardiovascular system, muscle damage, oxidative stress, and nerve conduction velocity is similar to regular exercise. ${ }^{8}$ However, serious side effects have been reported with BFR in healthy populations including venous thrombosis, rhabdomyolysis, and pulmonary emboli. ${ }^{9}$ Additional data are needed on the short- and long-term effects of BFR in patient populations. Clearly, the safety and efficacy of BFR need to be well established before widespread utilization should be considered.

\section{Acknowledgment}

The authors have no conflicts of interest to disclose.

\section{References}

1. Takarada Y, Takazawa H, Ishil N. Applications of vascular occlusion diminish disuse atrophy of knee extensors muscles. Med Sci Sports Exerc. 2000;32(12):2035-2039. PubMed ID: 11128848 doi:10.1097/ 00005768-200012000-00011

2. Ohta H, Kurosawa H, Ikeda H, Iwase Y, Satou N, Nakamura S. Lowload resistance muscular training with moderate restriction of blood flow after anterior cruciate ligament reconstruction. Acta Orthop Scand. 2017;74(1):62-68. doi:10.1080/00016470310013680

3. Pearson SJ, Hussain SR. A review on the mechanisms of blood-flow restriction resistance training-induced muscle hypertrophy. Sports Med. 2015;45(2):187-200. PubMed ID: 25249278 doi:10.1007/ s40279-014-0264-9

4. Iversen E, Rostad V, Larmo A. Intermittent blood flow restriction does not reduce atrophy following anterior cruciate ligament reconstruction. J Sport Health Sci. 2016;5(1):115-118. doi:10.1016/j.jshs. 2014.12.005

5. OCEBM Levels of Evidence Working Group. The Oxford 2011 Levels of Evidence. Oxford Centre for Evidence-Based Medicine. 2016. http://www.cebm.net/index.aspx?o=5653. Accessed December 14, 2017.

6. Moritani T, Sherman WM, Shibata W, Matsumoto T, Shinohara M. Oxygen availability and motor unit activity in humans. Eur J Appl Physiol Occup Physiol. 1992;64:552-556. doi:10.1007/BF00843767

7. Walloe L, Wesche J. Time course and magnitude of blood flow changes in the human quadriceps muscles during and following rhythmic exercise. J Physiol. 1988;405:257-273. PubMed ID: 3255792 doi:10.1113/jphysiol.1988.sp017332

8. Loenneke JP, Wilson JM, Wilson GJ, Pujol TJ, Bemben MG. Potential safety issues with blood flow restriction training. Scand J Med Sci Sports. 2011; 21(4):510-518. doi:10.1111/j.1600-0838.2010. 01290.x

9. Yasuda T, Ogasawara R, Sakamaki M, Ozaki H, Sato Y, Abe T. Combined effects of low-intensity blood flow restriction training and high-intensity resistance training on muscle strength and size. Eur $J$ Appl Physiol. 2011;111(10):2525-2533. PubMed ID: 21360203 doi: 10.1007/s00421-011-1873-8 